\title{
Fourth International Onnela Workshop Immunology and Diet - Towards the Prevention of Type 1 (insulin-dependent) Diabetes?
}

\author{
held in Janakkala, Finland, June 6-7, 1989
}

\author{
Report prepared by: H. K. Åkerblom \\ The Children's Hospital, 2nd Department of Pediatrics, University of Helsinki, Helsinki, Finland
}

Diet has traditionally been an important component in the treatment of diabetes in childhood, despite the unchallenged central role of insulin therapy. New concepts have emerged in recent years in this field, e.g. the more central role of carbohydrates in children's diet. Diet has come into focus in another respect regarding Type 1 (insulin-dependent) diabetes in recent years: many animal studies indicate that at least some dietary proteins may be involved in the aetiology and pathogenesis of Type 1 diabetes, and immunological mechanisms of action are thereby likely to be essential. These observations may be important with regard to Type 1 diabetes in children, therefore it was felt appropriate to review and discuss diet in relation to Type 1 diabetes with investigators working both in paediatric clinical practice and basic science laboratories. Finland is uniquely placed with regard to Type 1 diabetes in children in that the incidence of the disease is the highest in the world. Therefore, the interest in research on childhood diabetes in Finland has been and continues to be active.

The Fourth International Onnela Workshop was held in south Finland, Onnela, Janakkala, and was organized by the 2nd Department of Pediatrics, The Children's Hospital, University of Helsinki. Twenty investigators from five countries met for two days. The first session dealt with infant and childhood nutrition. J.K. Visakorpi (Department of Pediatrics, University of Tampere) described past and present trends in infant nutrition in Finland. The development of breast feeding during this century in Finland has been similar to many other industrialized countries. The lowest prevalence being in 1971 when only $6 \%$ of mothers breastfed until 6 months after the birth of the baby. At the end of the 1970's and especially during the 1980's a substantial increase in breast feeding took place reaching about $60 \%$ at 6 months. The substitute for breast milk in Finland was almost totally a home made cow's milk preparation with a high protein and casein content which was used until the beginning of the 1970 's. Thereafter, industrially prepared formulas have been used with low protein content $(1.5 \mathrm{~g} / 100 \mathrm{ml})$ and with modified whey/casein ratio as well as added vegetable oils. Present advice includes introducing solid foods after about three-four months of age. The continuation of exclusive breast feeding over six months is not recommended.

K. Launiala (Department of Pediatrics, University of Kuopio) summarized studies on milk consumption and lactose intolerance in children. The prevalence of selective late-onset hypolactasia (lactose malabsorption) in Finnish children and adolescents is $4 \%$ in $7-$ 11 year-olds, $9 \%$ in $12-15$ year-olds, $15 \%$ in $16-20$ year-olds and $17 \%$ in adults. The occurrence of symptoms is influenced by remaining lactase activity, amount of ingested lactose, functional stability of the colon, and variation in colonic microflora.

E. Isolauri (Department of Pediatrics, University of Tampere) reviewed the question of mucosal permeability of macromolecules during infancy. The gastrointestinal tract is a major port of entry for potentially antigenic macromolecules. An important adaptation of the gastrointestinal tract to the extrauterine environment is the development of a mucosal barrier against foreign proteins. When a system of non-immunologic and immunologic host defence mechanisms develops, the uptake of macromolecules decreases significantly. Under certain conditions (malnutrition, acute gastroenteritis, cow's milk allergy and prematurity) the normal barrier may be functionally or structurally altered leading to an increased absorption of macromolecules.

L. Madácsy (Postgraduate Medical Institute, Miskolc, Hungary) presented studies on the diet of normal Hungarian children, with particular reference to the consumption of milk. Nursing habits have changed recently in Hungary: the rate of breast-fed infants up to three months of age decreased from $42.6 \%$ in 1970 to $37.1 \%$ in 1982 . The average yearly consumption of milk in Hungary increased from 100 to $200 \mathrm{~kg} \cdot$ inhabitant ${ }^{-1}$. year $^{-1}$ between the years 1960 and 1987 , while $36 \%$ of the adult Hungarian population has intestinal lactase deficiency.

During the discussion it was emphasized that studies would be needed e.g. on the prevalence of lactase deficiency in diabetic children vs non-diabetic subjects in various populations.

The next session dealt with diet in the treatment of childhood Type 1 diabetes. L. Pinelli (Department of Pediatrics, University of Verona, Verona, Italy) described his experience of health education in the dietary treatment of Type 1 diabetes in children. In the past, diet for Type 1 diabetic patients was considered a means of achieving better glycaemic control, rather than good nutrition. This conventional diet, high in saturated fatty acids and in protein, is considered today harmful for the entire population. It is evident that diabetic children and adolescents have to be fed with particular care: a diet following the Recommended Daily Allowance may reduce the risk for major complications.

One session comprised papers on the genetic, immunological and environmental components of the aetiology of Type 1 diabetes. J.Nerup (Niels Steensens Hospital and Hagedorn Research Laboratory, Gentofte, Denmark) reviewed his and the Steno group's studies on the pathogenesis of Type 1 diabetes. The pathogenesis (i. e. the earliest events and mechanisms) leading to Beta cell destruction and Type 1 diabetes is MHC-non restricted, however, MHC associated and Beta cell specific. Interleukin-1 $\beta$ (IL-1 $\beta$ ) potentiated by tumour necrosis factor-alpha is the major Beta-cellcytotoxic effector molecule. This can be shown on isolated islets in vitro, in the intact perfused pancreas, and after in vivo treatment with IL-1 in normal rats. The Beta cell cytotoxic mechanism is unknown, but is suspected to be mediated by toxic free oxygen radicals. 
O.Pitkänen (and S.Andersson, M.Hallman and J.M.Martin, The Children's Hospital, University of Helsinki) reported some preliminary data on free oxygen radicals at the onset of Type 1 diabetes in $\mathrm{BB}$ rats. Lipid peroxidation was followed in four BB rats from 48 to 90 days of age. Expired pentane was measured by an adaptation to small rodents of a method described previously [1], in which $\mathrm{CO}_{2}$ and water is removed from the specimen before analysis by gas-chromatography. The basal rate of production of pentane was $1.6 \pm 0.6$ (SD) $\mathrm{pmol} \cdot 100 \mathrm{~g}^{-1} \cdot \mathrm{min}^{-1}$. All four rats became diabetic and, coincident with the development of overt diabetes (from three days before the appearance of glycosuria), pentane in expired air doubled $(p<0.02)$. The possibility that free oxygen radical-induced lipid peroxidation could be related to the pathological events leading to diabetes and its potential predictive value as an early indicator of the disease justifies further studies.

J.M. Martin (The Hospital for Sick Children, The Research Institute, Toronto, Canada, and Visiting Professor at The Children's Hospital, University of Helsinki) reviewed the question of cow's milk proteins as triggers of Type 1 diabetes. Dr. Martin and associates have earlier shown that in BB rats fed a diet with a complete mixture of synthetic amino-acids instead of proteins, the incidence of diabetes was reduced to $15 \%$ as compared with the $>50 \%$ incidence in the littermates fed either the same diet with $1 \%$ skim cowmilk powder added, or regular rat chow [2]. The protein restriction was effective only when applied during the weaning period and persisted even when a complete diet was introduced. Comparative analysis of the protein content in cow-/rat and human milks demonstrated a higher content of casein, beta-lactoglobulin and bovine serum albumin (BSA) in the first vs the others. Casein alone proved to be non-diabetogenic in the $\mathrm{BB}$ rat, while at the time of diabetes diagnosis, a significant increase in antibodies to BSA was found.

B.H.Robinson (and N.McKay, J.M.Martin, I.Sun and M. Glerum) (The Research Institute, The Hospital for Sick Children, Toronto, Canada) described the molecular basis for the interaction between dietary and genetic determinants. Western blotting or immunoprecipitation of rat islet Beta cell proteins with antibody to BSA reveals a cross-reacting protein of $M_{r} 67-69,000$. A region of BSA has been identified having homology to the beta-subunits of the MHC class II proteins $I_{a}, D Q$, and DR. The homology of the $\mathrm{DR} / \mathrm{DQ}$ allotypes found in the human population gave a strong correlation to the incidence of Type 1 diabetes. The peptide concerned produced antibodies when injected into rats that identified a $\mathrm{M}_{\mathrm{r}}=67,000$ protein. Antibodies to DR raised in rats also recognized a protein of identical $M_{r}$. This evidence supports the hypothesis that cow's milk proteins, particularly BSA are involved in the induction of Type 1 diabetes.

J.Ilonen (Department of Medical Microbiology, University of Oulu) spoke of the HLA-DQ polymorphism in the search for high risk individuals with Type 1 diabetes. Serological and especially DNA-hybridization studies strongly implicate that determinants in the HLA-DQ molecules are responsible for the association of Type 1 diabetes susceptibility with HLA-D gene area. Although the presence of aspartic acid at the position 57 in HLA-DQ beta-chain seems to be a powerful marker of protection in Caucasian populations, some Oriental haplotypes associated with Type 1 diabetes susceptibility remarkably seem to be Asp 57 positive. There are wide differences between the Type 1 diabetes risk carried by different Asp 57 negative haplotypes also in Western populations, but the combination of two different susceptibility markers in HLA-DQ area is a relatively specific marker for individuals at risk. This was also confirmed by us in restriction fragment length polymorphism studies of HLA-DQ beta-chain in Northern Finland, in which $51 \%$ of 61 Type 1 diabetes patients had a combination of $12 \mathrm{~kb}$ and $4 \mathrm{~kb}$ fragments compared to only $2 \%$ of 118 control subjects [3].

M.Knip (and J.Karjalainen, H.K. Åkerblom and the "Childhood Diabetes in Finland" Study Group) (Department of Pediatrics, University of Oulu) reported on islet cell-specific autoantibodies and metabolic risk markers in siblings of probands with Type 1 diabetes. Non-diabetic siblings in the nationwide "Childhood Diabetes in Finland" (DiMe)-study have been studied for conventional islet cell antibodies (ICA-IgG), complement-fixing ICA (CF-
ICA), insulin autoantibodies (IAA) and insulin secretory capacity as well as glucose metabolism by repeated intravenous glucose tolerance tests (IVGTT). Fifty-five/534 (10.3\%) were positive for ICAIgG, $46(8.6 \%)$ for CF-ICA and $11 / 589(1.9 \%)$ for IAA over a median observation period of 15 months. An IVGTT was performed in $52 \mathrm{ICA}$ and/or IAA positive siblings at least once. Nineteen siblings $(36.5 \%)$ had a low first phase insulin response (FPIR; $1+3 \mathrm{~min}<46 \mathrm{mU} / \mathrm{l}$ ) and/or a decreased glucose disappearance rate ( $\mathrm{kg}<1.30 \% / \mathrm{min})$. Over the observation period 12 of 693 siblings $(1.7 \%)$ presented with Type 1 diabetes. Six of 62 siblings $(9.7 \%)$ positive for ICA and/or IAA compared to 1 of 472 autoantibody negative siblings $(0.2 \% ; p<0.001)$ developed the disease. Among the risk markers evaluated, the combination of an abnormal FPIR and $\mathrm{K}_{\mathrm{g}}$ had the highest sensitivity $(100 \%)$ and positive predictive value ( 4 of $9 ; 44 \%$ ) for Type 1 diabetes.

S. Virtanen (and L.Räsänen and the DiMe Study Group) reported on preliminary dietary findings in the DiMe-study. Between 1 September 1986 and 1 September 1988531 0-14 year-old diabetic children and 521 3-19 year-old siblings participated in the DiMe nutrition substudy. In addition, dietary data from $870-6$-year-old control subjects were available. The duration of total and exclusive breast-feeding, the age when supplementary milk feeding or solid foods were introduced to the diet, consumption of milk products, coffee and tea, and the use of vitamin supplements by the diabetic patients, siblings and control subjects in three birth cohorts (1971$75,1976-80,1981-88$ ) were studied. No differences were found in any of these parameters in any birth cohort between diabetic subjects, siblings and control subjects. At present, a control group of children over 6 -years-old is being studied, and conclusions should only be drawn thereafter.

H.K. Åkerblom presented E. Savilahti's (The Children's Hospital, University of Helsinki) findings on cow's milk and beta-lactoglobulin antibodies in Type 1 diabetic children and their siblings in the DiMe-study. The material consisted of 302 newly diagnosed children with Type 1 diabetes and 226 non-diabetic siblings. The serum IgA class antibodies to cow's milk and beta-lactoglobulin of the former were higher $(p<0.005)$ than in the latter. When analysed by age groups, the trend to higher values in diabetic children remained. The mechanism(s) behind the elevation of these antibodies in diabetic children should be studied.

In the discussion the need to elucidate more the role of dietary components, e.g. that of certain proteins in the pathogenesis of Type 1 diabetes was stressed. The workshop finally discussed the general outline of intervention studies aimed at studying the impact of certain dietary proteins on the incidence of Type 1 diabetes in a high risk population.

Acknowledgements. The workshop was sponsored by Novo-Nordisk and the National Board of Health, Finland.

\section{References}

1. Pitkänen OM, Hallman M, Andersson SM (1989) Determination of Ethane and Pentane in Free Oxygen Radical-induced Lipid Peroxidation. Lipids 24: 157-159

2. Elliott RB, Martin JM (1984) Dietary protein: a trigger of insulindependent diabetes in the BB rat? Diabetologia 26:297-299

3. Reijonen H, Ilonen J, Knip M, Michelsen B, Åkerblom HK (1990) HLA-DQ beta-chain restriction fragment length polymorphism as a risk marker in Type 1 (insulin-dependent) diabetes: a Finnish family study. Diabetologia 33: 357-362

Prof. H. Åkerblom

$2^{\text {nd }}$ Department of Paediatrics

The Children's Hospital

University of Helsinki

SF-00290 Helsinki

Finland 